\section{EMBRYRIDDLE \\ Aeronautical University}

SCHOLARLY COMMONS
International Journal of Aviation, Aeronautics, and Aerospace

6-1-2016

\title{
User Interface Design Recommendations for Small Unmanned Aircraft Systems (sUAS)
}

\author{
Camilo Jimenez \\ University of Central Florida, camilo.j@knights.ucf.edu \\ Caitlin L. Faerevaag \\ University of Central Florida, faerevaag@knights.ucf.edu \\ Florian Jentsch \\ University of Central Florida, florian.jentsch@ucf.edu
}

Follow this and additional works at: https://commons.erau.edu/ijaaa

Part of the Aviation Commons, Cognition and Perception Commons, Cognitive Psychology Commons, and the Other Psychology Commons

\section{Scholarly Commons Citation \\ Jimenez, C., Faerevaag, C. L., \& Jentsch, F. (2016). User Interface Design Recommendations for Small Unmanned Aircraft Systems (sUAS). International Journal of Aviation, Aeronautics, and Aerospace, 3(2). https://doi.org/10.15394/ijaaa.2016.1118}

This Article is brought to you for free and open access by the Journals at Scholarly Commons. It has been accepted for inclusion in International Journal of Aviation, Aeronautics, and Aerospace by an authorized administrator of Scholarly Commons. For more information, please contact commons@erau.edu. 


\section{User Interface Design Recommendations for Small Unmanned Aircraft Systems}

(sUAS)

\section{Cover Page Footnote}

ACKNOWLEDGMENTS The views and opinions expressed in this paper are those of the authors and do not necessarily represent those of the Federal Aviation Administration (FAA) or of the institutions with which the authors are affiliated. 
In recent years, there has been an expansion in the use of small Unmanned Aircraft Systems (sUAS) for recreational and commercial purposes. The Federal Aviation Administration (FAA) estimated that nearly 1.6 million of these systems are intended for recreational purposes, and would be sold throughout 2015 (FAA, 2015a). During the first month after the FAA's online registry system for sUAS went live, nearly 300,000 sUAS owners had registered (FAA, 2016), indicating that at least the same number of sUAS were being used or intended to be used within the National Airspace (NAS). As the FAA begins to develop regulations for the recreational, commercial, and public operations use of sUAS (FAA, 2013), the number and variety of sUAS is expected to rise. Despite the growth of sUAS usage and an attempt by the FAA to regulate the operation of these systems, there are no clear guidelines explaining the type of information that should be available to sUAS operators while flying in the NAS.

Equally important is the need to create guidelines that aid sUAS manufacturers in developing visual user interfaces (UI) that comply with design principles that enhance the flying experience of sUAS users. This could be achieved by providing information in a way that helps operators maintain Situational Awareness (SA) of their aircraft and surroundings, thereby reducing the chances of them violating regulations applicable to sUAS. This is important because the intended user of such systems may not yet be familiar with the aeronautical rules and regulations that other certified pilots, operators, and traffic controllers are, specifically in relation to the use of airspace. sUAS operators, nonetheless, must become familiar with these rules and regulations because they will be sharing an already complex airspace; not only with other small unmanned aircraft operators, but also with other manned aircraft.

The purpose of this paper is threefold. First, we want to remind the aviation community, in addition to researchers and practitioners unfamiliar with sUAS, of the impact that these small unmanned aircraft are having (and will continue to have) on the NAS. Second, we want to expose researchers and practitioners within the aviation community to the FAA's (2015b) Small UAS Notice of Proposed Rulemaking (NPRM), which intends to add a new part 107 to title 14 CFR that regulates the operation of sUAS in the NAS. In this sense, we believe it is important to understand the rules and regulations being proposed in the Small UAS NPRM in order to initiate a dialogue among different aviation stakeholders, with the intent of identifying the specific types of information that should be available to sUAS operators while flying in the NAS. Third, we want to present researchers and designers interested in the development of sUAS visual interfaces with a set of recommendations that could facilitate and support the design of intuitive interfaces. As a result, the recommendations could enhance 
operators' SA of the legal status of their flight (e.g., whether or not they are operating within airspace boundaries). The overall objective is to design UI that allow operators unfamiliar with the intricacies of our NAS to safely operate within allowed airspace, and to discourage or prohibit the unlawful use of these systems; this will serve to increase safety and reduce the number of incidents and accidents involving sUAS.

\section{Issues Associated with the Integration of sUAS into the NAS}

Incidents and accidents caused by human error and equipment failure are much higher for military UAS than for manned aircraft (Williams, 2004; McCarley \& Wickens, 2004). We expect this to be true for civilian UAS operation as well, but on a much larger scale due to the large number of sUAS entering the NAS. According to the FAA (2015c), the number of unmanned aircraft sightings and close encounters reported by pilots has dramatically increased. Some of these sightings have been reported at altitudes in which the operation of such aircraft is prohibited (over 500 feet above ground level [AGL] and as high as 10,000 feet). Gettinger and Michel (2015) reported that between December 2013 and September 2015 nearly 35\% of the 921 incidents involving manned and unmanned aircraft in the NAS were categorized as close encounters (i.e., within 500 feet of a manned aircraft). The issue of integrating sUAS into the NAS presents a unique set of challenges to the FAA and the design and research community. While developing regulations and operational limitations for sUAS, the FAA must consider the population of users that will be entering an already crowded airspace.

Many sUAS operators, especially recreational users, will have little understanding or appreciation for existing regulations and principles of aviation. Unauthorized or unsafe use of sUAS can and have caused an immediate threat to commercial flights in congested airspaces near major airports. They have even been known to interfere with emergency operations such as wildfire suppression (FAA, 2015a). These instances resulted in wasted federal and municipal resources and threatened life and property. For example, the FAA (2015a) reported that in June of 2015, a number of sUAS interfered with an aerial firefighting operation near Big Bear City, CA. The aborted mission was estimated to have cost between $\$ 10,000$ and $\$ 15,000$ in operational costs alone. In this instance, the FAA had issued a temporary flight restriction (TFR) in the area surrounding the fire. The restriction was either not received, not understood, or not respected by sUAS operators. This resulted in hazardous conditions for the firefighting tanker planes and the nearby community. Unfortunately, non-compliance with TFR is a 
Jimenez et al.: U ser Interface Design Recommendations for sU AS

seemingly inherent problem of introducing a large number of novice users into the NAS.

As the number of incidents is expected to grow with the number of sUAS in the NAS, many anticipatory solutions are being proposed. They include, but are not limited to, creating a database of sUAS owners and outfitting sUAS with safety systems that would prevent them from coming within close proximity to an airport ( 5 miles, unless previous authorization has been given by an air traffic controller) or flying over 500 feet AGL. Additionally, the FAA and other members of the pilot and aeromodelling community are working diligently to institute a solution that informs sUAS owners of the regulations and limitations imposed on such systems.

There is a multitude of initiatives currently being proposed that would keep sUAS operators from operating outside of their allotted airspace. However, there is no known literature that mentions the benefits of designing visual UI that would aid sUAS operators in maintaining established NAS limits or support the development of standardized UI visual design guidelines for sUAS manufacturers. This has led to a number of sUAS manufacturers putting products on the market that not only demonstrate a lack of adherence to human-centered design principles, but also contain a plethora of incongruous and non-intuitive UI.

\section{The Importance of Developing User-Friendly, Standardized Instruments for sUAS Operations}

In the past, the aviation community has encountered issues associated with non-standardized controls and display design. During WWII, training military pilots was unnecessarily dangerous, costly, and time-consuming, due in part to the unstandardized design of cockpits (Mark, Warm, \& Huston, 1987). Consequently, the Army alleviated these training issues by empirically designing and testing intuitive, user-friendly controls and displays that would become the standard for all aircraft, regardless of manufacturer. This allowed pilots to train in one aircraft and fly another. As a result, the standardization of display designs mitigated the problems associated with negative transfer of training and reduced the unnecessarily burdensome cognitive load brought about by haphazardly designed controls and displays. In addition to this challenge, the control stations for these systems do not currently conform to any standard of design. sUAS design variety is also reflected in the variety of control stations used to operate these systems. Some control stations provide less visual information to the operator, while others integrate phones/tablets to provide sUAS status information (e.g., altitude, distance from the operator, battery life, and first-person view video feedback from sUAS). Some entities have recognized the importance of standardizing control 
stations. For example, the U.S. Army (2016) announced that it is working towards a universal control station that would, among other things, facilitate operator training and allow them to fly more than one type of UAS. As during WWII, the field of Human Factors is uniquely equipped to help develop a design standard that would enable operators to safely and intuitively control sUAS while facilitating a smoother integration of sUAS into the NAS. In order to start developing standardized visual display indicators for sUAS, we first need to understand the proposed regulations and limitations for this type of system.

\section{Proposed Restrictions on sUAS Operation}

On February 15, 2015, the FAA released the Small UAS NPRM, which outlined the proposed provisions that would be included in Part 107. This NPRM discussed potential limitations and operator responsibilities involving sUAS. Table 1 shows some of the proposed operational limitations for sUAS including maximum airspeed, altitude, and the required distance between the operator and the sUAS. This section provides an overview of the most important aspects of the sUAS NPRM provisions that could, in principle, help the interested researcher or practitioner understand the type of information that an operator should have before and during flight. We present this information with the intent of starting a discussion within the aviation community about the design of visual indications presented on a display designed for this type of system.

Some other important aspects of the sUAS NPRM involve operational limitations, and include: conduct of aircraft and control station pre-flight inspections, prohibition of sUAS operation in the vicinity of non-involved persons, and prohibition of sUAS operation during a time of physical or mental impairment of the operator.

\section{The Question Raised by the NPRM}

The NPRM raises several questions that should be addressed by the research community, manufacturers, and the FAA. For example, the FAA proposed a minimum visibility but did not address other weather conditions that may interfere with operations, such as high sustained winds, gusting winds, or winds aloft. The relatively small size of these aircraft will make them difficult to operate and control under strong wind conditions. The wind will also impact the relative ground speed, depending on whether the wind conditions create a headwind or a tailwind. However, the NPRM does not include a maximum groundspeed. The wind will also impact range, as an sUAS flying with a tailwind away from the operator will require more battery life to make it back to the origin 
point than it required to reach its maximum distance from the operator. An inexperienced operator may not account for this, resulting in battery exhaustion in mid-flight.

Table 1

Proposed operational limitations for sUAS. Adapted from FAA (2015c).

Regulation

Limitation

\begin{tabular}{|c|c|}
\hline Maximum weight & 55 lbs. \\
\hline Maximum airspeed & $100 \mathrm{mph}$ \\
\hline Maximum altitude & 500 feet above ground level \\
\hline $\begin{array}{l}\text { Maximum allowed distance } \\
\text { from operator }\end{array}$ & $\begin{array}{l}\text { Must maintain a visual line of sight (VLOS) } \\
\text { with vision unaided, except for the use of } \\
\text { corrective lenses }\end{array}$ \\
\hline Minimum weather visibility & 3 miles from operator \\
\hline Allowed area of operation: & $\begin{array}{l}\text { Class } A \text { : Prohibited } \\
\text { Class } B, C, D \text {, and } E \text { : Air traffic controller } \\
\text { (ATC) authorization needed } \\
\text { Class } G: \text { No ATC authorization needed }\end{array}$ \\
\hline Time restrictions & From official sunrise to official sunset \\
\hline $\begin{array}{l}\text { Number of operators per } \\
\text { sUAS }\end{array}$ & $\begin{array}{l}\text { One operator per sUAS } \\
\text { Operator may use a visual observer }\end{array}$ \\
\hline
\end{tabular}

Another question that must be addressed involves how Notices to Airmen (NOTAMs) and meteorological information (e.g., METARs) should be relayed to operators. In manned flight, pilots rely on METAR information, which provides visibility and wind speed. However, METAR information is only relevant and available in close proximity to an airfield, where the operation of sUAS will be restricted. Relaying NOTAM information is crucial to safe sUAS operation, especially in the case of a TFR. Equally important is the need for clarification from the FAA on the following topics: required pre-flight inspection procedures, sUAS technical and mechanical airworthiness standards, and courses of action for eliminating airworthiness flaws for future flight eligibility (e.g., guidelines on what to do if one or more visual displays/indicators are malfunctioning, and what minimum required operational equipment and indicators are needed for the sUAS to be considered airworthy). 
The proposed regulations do not address any aspect of information availability necessary for sUAS operations or any interface design recommendations for this type of system. Consequently, we propose a series of recommendations that will aid in the design of visual displays for sUAS. Identifying the type of information that should be available to users during different stages of flight and designing optimal display systems could significantly aid in the effort of safely integrating sUAS operations into the NAS. Reaching a consensus among members of the aviation community on the type of information necessary for operations and how it should be presented to operators could help in the development of standardized displays for sUAS. Standardized displays are a norm in manned aircraft operations (e.g., altitude, airspeed, and attitude indicators); it follows that a standardized set of indicators should also be developed for sUAS. This is especially important if sUAS are to be permanently integrated into the NAS.

In addition, a standardized set of visual indicators could also facilitate the educational efforts to train sUAS operators by teaching them: what the information presented to them means, why it is relevant to their operations, and how to effectively apply aircraft controls based on the information they see on the display. The intent behind presenting this set of recommendations to the aviation community is to start a conversation about the importance of a) the type of information that should be available to users during sUAS operations, and b) how to design usable visual displays that could facilitate the safe operation of these systems. Considering the restrictions in the NPRM and the questions raised by it, we suggest the following recommendations be taken into consideration.

\section{Design Recommendations for sUAS Controls and Visual Displays}

\section{User's Mental Expectations}

A well-designed visual display should match the mental expectations of the operator. Tlauka (2004) explained that designing a visually and cognitively user-friendly control and display interface could improve operator response time and increase user satisfaction. In other words, a display should aid operators by enhancing their response to a stimulus, reducing the stimulus-response time by being in accordance with the controls needed to perform the task. Having a display that closely matches the depicted environment could significantly reduce the operator's workload. The information displayed should move in a direction consistent with the operator's mental model (e.g., a vertically oriented altitude indicator representing the vertical plane). Korblum, Hasbroucq, and Osman (1990) proposed what they called a dimensional overlap model. This model shows that when a stimulus-response ensemble shares a number of characteristics, the 
Jimenez et al.: U ser Interface Design Recommendations for sU AS

stimulus will activate an automatic response due to the features shared by both the stimulus set and the response set. The automatic response creates a reduction in reaction times, and increases the probability of a correct response. When the stimulus sets and the response sets do not share characteristics, responses may be slower and more prone to error.

Eimer (1995) performed a series of experiments in which he found that participants' reaction times were faster when a cue (arrow) indicated in which direction a target letter would appear on a computer screen. When the cue did not indicate in which direction the letter would appear, participant reaction times were slower. These series of experiments indicated that cues that effectively alerted the participant of the direction in which the letter would appear on the screen elicited automatic responses. These findings were in accordance with Kornblum et al.'s (1990) dimensional overlap model, and illustrate the need for designing interfaces consistent with the mental models and expectations of the user. For example, an auditory or visual alert advising the operator to descend (due to having reached maximum allowed altitude) should be accompanied by controls that meet the mental expectations of the operator (e.g., pressing down a lever, or a button shaped like a downward arrow), allowing him/her to rapidly take action without major cognitive processing on how to apply controls to respond to the altitude alert. The congruency of alert (descend), control (press lever down), and mental expectation (pressing down the lever would make the sUAS descend) can elicit rapid, automatic responses that are less prone to error.

\section{The Need to Distribute Attention}

UAS operators need to distribute attention to many different kinds of information (e.g., speed, altitude, and other aircraft in the vicinity). Zhang (1997) referred to distributed cognitive tasks, such as flying an aircraft, as tasks that require operators to process information coming from the external environment and integrate it with information retrieved from internal interpretations in a dynamic manner. In this sense, Zhang argued that external representations are picked up through perceptual processes, while internal representations come from cognitive processes that involve schemas, mental images, and neural networks. To perform distributed cognitive tasks, it is necessary that the information from internal and external representations is integrated and exchanged, not only in a dynamic manner, but in an integrative way. It is important to understand that visual displays for complex tasks should allow operators to switch between focused attention and divided attention when needed. Parasuraman and Davies (1984) discussed the impact that these two types of attention have on performance. While focused attention allows operators to fixate and process 
certain characteristics of a display, divided attention allows operators to integrate the information perceived from different sources. The goal of integrating divided attention and complex tasks in properly designed displays is to create subsystems (individual indicators) that allow operators to integrate these sources of information while maintaining efficient levels of performance (Parasuraman \& Davies, 1984; Zhang, 1997; Tlauka, 2004). One of the most common examples in aviation is the layout of what's commonly known as the "six pack" (i.e., Airspeed, Attitude, Altimeter, Vertical Speed, Heading, and Turn coordinator indicators). While each indicator provides unique information to be processed individually, this layout allows pilots to crosscheck each instrument and integrate information from individual indicators to assess aircraft performance. For instance, if a pilot wants to know if the aircraft is descending at a constant rate in a particular heading, he or she should crosscheck the information provided by the airspeed, vertical speed, altitude, and heading indicators. Similarly, with sUAS, visual display layouts should consider how individual pieces of information relate to each other, and considerations should be taken to allow for information integration and processing.

\section{Data Extraction}

Bennet and Flach (1992) explained that in integrated tasks, attention must be distributed among different information sources that need to be considered in order to reach a decision. Consequently, it is important that both the type of information transmitted to the operator and the information that is presented should be considered when designing sUAS displays. Woods (1991) discussed the importance of designing for both data availability and information extraction. Systems that only considering data availability usually force the operator to maintain the data in their memory, while simultaneously forcing them to retrieve information from their long-term memory, causing an exhaustion of limited cognitive resources. Thus, displays that replace memory with perception are considered to improve performance because they do not use the cognitive resources involved in information processing (Bennett \& Flach, 1992). Regarding sUAS design, the controller should present data relevant to operation in a way that does not force operators to rely on long-term memory (to the extent possible). Additionally, the design should avoid requiring any unnecessary cognitive work like mental calculations. For example, to avoid accidents in which battery exhaustion causes the sUAS to fall to the ground, it would be beneficial to display battery life in a way that supports efficient use of the information. In this case, the battery life of the system is not as important as the expected range. By displaying expected range and distance from the operator (controller), several mental 
calculations would be removed, reducing the amount of time and work it takes for the operator to determine if they should end the flight to avoid an accident.

\section{Redundancy}

The operation of an sUAS is a complex task. The user is required to operate the system, monitor the system, and operate related systems (e.g., cameras). While performing these tasks, operators are also required to maintain visual contact with the sUAS. It is important to design displays that minimize the amount of heads-down time. A good display should be able to provide information in more than one way. Information is most effective when different modalities are used to get the operator's attention (i.e. a combination of visual and aural sources of information) (Wickens, Gordon, \& Liu, 2004). In addition to reducing the need for the operator to exclusively rely on visual displays, aural and/or haptic displays could supplement visual information in alerting the operator of important information without breaking visual contact with the sUAS. For example, an ideal altimeter design would provide the operator through haptics the following data: when the sUAS approaches restricted airspace (e.g. the 500 feet AGL limit), and then give clear aural instructions (e.g. "descend") to correct the error. One issue unique to UAS operation is the absence or degradation of multi-sensory feedback inherent in manned flight: movement, vibrations, sounds, etc. This relative lack of sensory feedback results in loss of SA (Lam, Mulder, \& Van Paassen, 2007). Lam et al. (2007) found that haptic warnings were effective in assisting teleoperators in avoiding collisions when visual information was insufficient. Haptic and aural displays have the potential to ameliorate both of the issues associated with external pilot teleoperation by reducing mental workload and supplementing insufficient visual information (Lam, et al., 2007; Lam, Delannoy, Mulder, \& Van Paassen, 2005).

\section{Usability as a Way to Increase Trust in the Displayed Information}

Designing usable displays is critical in sUAS operations, because using poorly designed interfaces can endanger operators, property, other aircraft, and the general public. sUAS operators will rely on displays to inform them of speed, altitude, distance, battery life, etc. This information must be reliable and easily interpreted. If sUAS are going to be safely integrated into the NAS, displays must be designed and tested to the highest standard of usability. It is important that sUAS displays not only allow users to comply with regulations, but also provide information in a user-friendly manner if they are to be accepted by the user. Userfriendly controls and displays are more likely to be trusted and utilized in an

appropriate manner. Lee and Nass (2010) explained that trust in relation to 
technological systems can be defined as the level of confidence the operator has in the system, particularly when the achievement of a goal in an uncertain situation is necessary.

Training users/operators on how to use systems low in usability tends to be complicated, expensive and sometimes futile, as in the case of the WWII pilots discussed earlier. As a consequence of poor design and low levels of usability, the system will more likely be misused or disused (Maguire, 2001; Chamorro-Koc et al., 2009; Parasuraman \& Riley, 1997). In sUAS interface design, this is particularly risky, due to the threat to the NAS and persons/property on the ground if the systems are misused or alerts are ignored. For example, in a study conducted by Acemyan and Kortum (2012), results showed that higher scores of perceived usability of everyday technological devices translated into higher levels of trust by the user. This relationship is even stronger when the user is given no choice and is required to use a specific system. There are many circumstances in which operators have no choice but to interact with the tools that have been provided to them to execute a task, but this is not always the case in sUAS operation. A number of manufacturers are producing sUAS for commercial and recreational use, and each manufacturer has the liberty of designing displays and controls in a manner they think will appeal to their target market. Some displays are customizable, allowing customers to personalize their display, further increasing the variability of sUAS display design. Therefore, creating usable displays that provide reliable, easily understood information will increase the user's trust in the display, reducing the chances of underuse and misuse.

A note on usability testing: in designing for sUAS, the goal should be to design controls and displays that operators perceive as usable to increase the user's level of trust in the system and the information available to them. This will encourage appropriate use and reliance on the system. It is important that every interface be tested with the end user in mind. Usability studies are of great importance because it is essential to understand the interaction between humans and systems (Acemyan \& Kortum, 2012). Usability studies focus on the assessment of the difficulties that users encounter when interacting with products in applied settings, while simultaneously trying to find ways to improve the manner in which users interact with products (Chamorro-Koc, Popovic \& Emmison, 2009). Although researchers, designers, and engineers design displays under the consideration of how these designs will be used by the operators, it is also important to test and iterate designs using the end user's feedback. When a display has been designed, optimization is only achievable during usability testing. 


\section{Conclusions}

The FAA is currently developing and structuring sUAS operating restrictions. This paper outlines the gaps in the information being used to craft those restrictions. We intend for the FAA to address the questions raised by the gaps, as outlined in this paper, in their proposed restrictions. In the intervening period, it is imperative that the research and design community works to determine what information will best support the sUAS user in safe and legal operations in the NAS, then develop appropriate ways to relay this information to users in accordance with good principles of design. Several examples and suggestions have been expressed in this paper; however, these display recommendations must be tested empirically in the context of sUAS display design, to determine the optimal way of displaying information in the most userfriendly way possible. While the FAA is still developing regulations, the NPRM gives researchers and designers an idea of the type of information and capabilities an sUAS operator will need in order to safely operate in accordance with future regulations. In the future, researchers and designers should account for these restrictions when determining how to display information.

As the availability and popularity of sUAS increases, an accepted standard will emerge for sUAS interface design. This standard should be based on empirical evidence and usability research, rather than a unilateral decision by a handful of manufacturers. As stated by Hall, Shattuck and Bennett (2012), "The ultimate goal is to design interfaces that (a) are tailored to specific work demands, (b) leverage the powerful perception-action skills of the human, and (c) use powerful interface technologies wisely" (p. 166).

A standard for sUAS displays would benefit sUAS users and the aviation community as a whole. It will simplify the training process, and facilitate communication between sUAS operators and the FAA. If designs are standardized, users will be able to transfer their training between devices without learning a new system, which will potential accidents resulting from negative transfer. Displays should also facilitate communication between users and authorities, allowing the FAA or ATC to issue warnings and flight restrictions directly to operators in real time.

The recommendations described above are intended to support the FAA with their current efforts, and support the development of future guidelines for sUAS designers and manufacturers. As such, based on our recommendations, we deem it important that a standardized set of guidelines be compiled for manufacturers of sUAS, and that the guidelines are informed by display design 
principles and usability research. To the extent that display design affects the usability of a device, optimizing and standardizing displays would encourage operators to trust in the reliability of the information they receive, and safely operate within the intended airspace. In short, standardization would increase safety for users, other air traffic, and the general public. The first priority should be to identify the type of information necessary for safe sUAS operation, then develop an appropriate way to display this information according to good design principles. Researchers, designers, and manufacturers should immediately begin the process of designing displays and tools that facilitate safe sUAS operation in order to stymie the influx of less-than-optimal designs deployed in the NAS.

\section{Acknowledgments}

The views and opinions expressed in this paper are those of the authors and do not necessarily represent those of the Federal Aviation Administration (FAA) or of the institutions with which the authors are affiliated. 


\section{References}

Acemyan, C. Z., \& Kortum, P. (2012). The relationship between trust and usability in systems. Proceedings of the Human Factors and Ergonomics Society 56 ${ }^{\text {th }}$ Annual Meeting, 56(1), 1842 - 1846. doi: $10.1177 / 1071181312561371$

Bennett, K. B., \& Flach, J. M. (1992). Graphical displays: Implications for divided attention, focused attention, and problem-solving. Human Factors: The Journal of the Human Factors and Ergonomics Society, 34(5), 513 - 533. doi: 10.1177/001872089203400502

Chamorro-Koc, M., Popovic, V., \& Emmison, M. (2009). Human experience and product usability: Principles to assist the design of user-product interactions. Applied Ergonomics, 40, 648 - 656. doi: 10.1016/j.apergo.2008.05.004

Eimer, M. (1995). Stimulus-response compatibility and automatic response activation: Evidence from psychophysiological studies. Journal of Experimental Psychology: Human Perception and Performance, 21(4), 837 854. doi: 10.1037/0096-1523.21.4.837

Federal Aviation Administration. (2013). Integration of civil Unmanned Aircraft Systems (UAS) in the National Airspace System (NAS) roadmap. Retrieved from http://www.faa.gov/uas/media/uas_roadmap_2013.pdf

Federal Aviation Administration. (2015a). Registration and marking requirements for small unmanned aircraft. Retrieved from https://www.federalregister.gov/articles/2015/12/16/2015-31750/registrationand-marking-requirements-for-small-unmanned-aircraft\#h-9

Federal Aviation Administration. (2015b). Operation and certification of small unmanned aircraft systems. Retrieved from https://www.faa.gov/regulations_policies/rulemaking/recently_published/medi a/2120-AJ60_NPRM_2-15-2015_joint_signature.pdf

Federal Aviation Administration. (2015c). Pilot reports of close calls with drones soar in 2015. Retrieved from https://www.faa.gov/news/updates/?newsId=83445 
Federal Aviation Administration. (2016). FAA registered nearly 300,000 unmanned aircraft owners. Retrieved from https://www.faa.gov/news/press_releases/news_story.cfm?newsId=19914

Gettinger, D., Michel, A.H. (2015). Drone sightings and close encounters: An analysis. Retrieved from http://dronecenter.bard.edu/drone-sightings-andclose-encounters

Hall, D. S., Shattuck, L. G., \& Bennett, K. B. (2012). Evaluation of an ecological interface design for military command and control. Journal of Cognitive Engineering and Decision Making, 6(2), 165 - 193. doi: http://dx.doi.org/10.1177/1555343412440696

Kornblum, S., Hasbroucq, T., \& Osman, A. (1990). Dimensional overlap: cognitive basis for stimulus-response compatibility--a model and taxonomy. Psychological review, 97(2), 253 - 270. doi: 10.1037/0033-295X.97.2.253

Lam, T. M., Delannoy, M., Mulder, M., \& Van Paassen, M. M. (2005). Effects of haptic feedback in the teleoperation of an unmanned aerial vehicle. Proceedings of the 13th International Symposium on Aviation Psychology, pp. 339-344.

Lam, T. M., Mulder, M., \& Van Paassen, M. M. (2007). Haptic interface for UAV collision avoidance. The International Journal of Aviation Psychology, 17(2), 167-195. doi: 10.1080/10508410701328649

Lee, J. R., \& Nass, C. I. (2010) Trust in computers: The computers-are-socialactors (CASA) paradigm and trustworthiness perception in human-computer communication. In D. Latusek, \& A. Gerbais (Eds.), Trust and technology in a ubiquitous modern environment: Theoretical and methodological perspectives (pp. 1 -15). Hershey, PA: Information Science Reference.

Maguire, M. (2001). Methods to support human-centred design. International journal of human-computer studies, 55(4), 587 - 634. doi:

10.1006/ijhc.2001.0503

Mark, L. S., Warm, J. S., \& Huston, R. L. (1987). Ergonomics and human factors: Recent research. New York: Springer-Verlag. 
McCarley, J. S., \& Wickens, C. D. (2004). Human factors concerns in UAV flight. FAA's FY04 Human Factors Research and Engineering Division Program. Washington, DC: FAA.

Parasuraman, R., \& Davies, D. R. (Eds.). (1984). Varieties of attention (Vol. 40, pp. $47-52)$. New York: Academic Press.

Parasuraman, R., \& Riley, V. (1997). Humans and automation: Use, misuse, disuse, abuse. Human Factors: The Journal of the Human Factors and Ergonomics Society, 39(2), 230-253. doi: 10.1518/001872097778543886

Tlauka, M. (2004). Display-control compatibility: The relationship between performance and judgments of performance. Ergonomics, 47(3), 281 - 295. doi: 10.1080/0014013031000157931

U.S. Army. (2016). Army to go universal with UAS operator training. Retrieved from http://www.army.mil/article/161492/ Army_to_go_universal_with_UAS_operator_training/

Wickens, C. D., Gordon, S.E., Liu, Y. (2004). An introduction to human factors engineering. Reading, MA: Addison Wesley.

Williams, K. W. (2004). A summary of unmanned aircraft/incident data: Human factors implications. FAA Technical Report, DOT/FAA/AM-04-24, Oklahoma City, OK: FAA.

Woods, D. D. (1991). The cognitive engineering of problem representations. In G.R.S. Weir and J.L. Alty (Eds.), Human-computer interaction and complex systems (pp. 169 -188). London: Academic.

Zhang, J. (1997). Distributed representation as a principle for the analysis of cockpit information displays. The International Journal of Aviation Psychology, 7(2), 105 - 121. doi: 10.1207/s15327108ijap0702_1 